\title{
PRODUCTION OF HELIUM BY STELLAR EVOLUTION
}

\section{R. KIPPENHAHN}

Universitäts-Sternwarte Göttingen, Göttingen, D.B.R.

In order to maintain the luminosity of the Galaxy $\frac{1}{3}$ of a solar mass of hydrogen has to be transformed into helium every year. This rate of production is too small by a factor 10 or 20 in order to give a helium content of $Y=0.3-04$. within the age of the galaxy if the mass fraction $Y$ of helium was zero at the beginning. The situation is even worse if the destruction of helium by helium burning is taken into account. In his review paper Tayler (1967) came already to this conclusion. I shall discuss the problem here using more recent model calculations, but we shall come up with the same result.

\section{Helium Production and Destruction}

If the interstellar gas forms stars of different masses, the objects with $M<0.1 M_{\odot}$ will never reach the temperature of hydrogen burning and therefore no helium will be produced in their interiors. Stars in the mass range $0.1 M_{\odot}<M<0.5 M_{\odot}$ reach the stage of hydrogen burning, but after the exhaustion of central hydrogen in the subsequent phase of central contraction the objects become degenerate and will cool off before they reach the temperature of helium burning (Hayashi et al., 1962). These stars are the really good helium generators since they form helium but do not destroy it. However, these stars of low mass can be neglected for the helium production in our Galaxy since due to the low luminosity their rate of production is rather small. In the mass range $0.5 M_{\odot}<M<1.5 M_{\odot}$ a helium core is formed on the main sequence. This core grows in mass but contracts slowly until the temperature of helium burning is reached and the destruction of helium starts. The most extensively investigated case is that of $1.3 M_{\odot}$ which might be typical for the whole mass range. Because of the degeneracy of the helium, the core helium burning sets in violently (helium flash). Because of the neutrino losses, helium is not ignited in the center but in a shell. The computations (Thomas, 1967) indicate that first the innermost $40 \%$ of the mass of the star is transformed from helium into higher elements. Then the helium burning shell eats outwards approaching closer and closer the hydrogen burning shell. This is very typical for practically all cases of advanced stellar evolution. Sometimes the hydrogen burning shell has been extinguished with the onset of helium burning, but when the helium burning shell approaches the hydrogen rich envelope the temperature rises and hydrogen burning starts again. One then has two shells, both eating outwards. The mass in between these two shells contains only a few percent of the mass of the star. Only the mass between these shells is pure helium which could enrich the interstellar material if the star's mass somehow is ejected into the interstellar space.

For the more massive stars we take as an example the case of $5 M_{\odot}$. Here the 
helium produced in the hydrogen burning phase is distributed throughout the central core by convection. Then a hydrogen burning shell is formed surrounding a helium core in the center of which helium will be ignited. At the very end again one obtains two shells eating outwards. It can happen that with the onset of helium burning, when the star is a red giant or supergiant, the convection in the outer envelope penetrates deeply inwards and even reaches the helium core, mixing helium (which has been formed during the main sequence stage) outwards into the envelope (Kippenhahn et al., 1965, see especially Figure 2 of that paper near the age of $8 \times 10^{7} \mathrm{yr}$ ). This is the best that could happen to the helium since in later phases almost all the helium in the interior will be used up by the helium burning shell. Indeed, in the case we are discussing here due to convective mixing the helium content of the outer layers is increased by $\Delta Y=0.06$. In the subsequent phases the mass $\Delta M$ of helium between the two shells is $\Delta M=6 \times 10^{-4} M$ (Weigert, 1966). It therefore seems that it can be neglected for the galactic helium production.

An exception are stars of $15 M_{\odot}$ or higher (Paczynski, private communication). In these stars the higher nuclear reactions in the center occur so fast that the helium burning shell had not enough time to approach the hydrogen burning shell and in these cases there might be not enough time to destroy most of the helium in the star before an appreciable amount of the stellar mass is blown into space.

\section{Mass Loss}

There are several mechanisms which might produce significant mass loss: stellar wind, dynamical instability in the red giant region (Lucy, 1967; Roxburgh, 1967; Paczynski and Ziolkowski, 1968), outgoing mass from pulsating stars. But these mechanisms are not yet worked out sufficiently well in order to predict how much mass will go into the interstellar space and during which phase of evolution the mass loss takes place. On the other hand it is also difficult to observe from the outside whether an evolved star has lost mass from the surface. If one peels ofi mass from the surface of an evolved star the envelope immediately readjusts within its KelvinHelmholtz time scale and the star resumes the old radius. This behaviour has been thoroughly investigated in the calculations of mass exchange in close binary systems (Kippenhahn et al., 1967).

From the pulsation theory it has been concluded that stars crossing the cepheid strip have lost half of the main sequence mass (Christy, 1966, 1968; Stobie, 1969). But now it seems that uncertainties in the stellar opacity (which influence more the pulsation calculations than the calculations of evolutionary tracks) could be made responsible for the discrepancy between normal stellar evolution theory (which assumes no mass loss during the helium burning stages) and the non-linear pulsation calculations. A very strong argument against mass loss in these very early post main sequence stages comes from investigations by Lauterborn et al. (in press). They dealt with the evolutionary track of a star of $5 M_{\odot}$ which showed loops in the red giant stage with several slow crossings of the cepheid strip. It turned out that if more than 
$5 \%$ of the mass of the star were taken off from the envelope the loops disappeared and therefore there were no slow crossings any more of the instability strip. Since cepheids exist there can be no appreciable mass loss during that phase! But even if such a star looses half of its mass the helium shell would still be safe in the deep interior.

Even if the Lucy-Paczynski-Roxburgh-Ziolkowski mechanism would work only the hydrogen rich envelope would go into the interstellar space. We therefore conclude that during the phases of evolution which we can now follow, the situation is not favourable for an enrichment of the galactic helium content by the helium produced in stars.

But one should be careful with such a conclusion. The $\mathrm{R} \mathrm{Cr}$ B stars seem to be a counterexample. Due to the infrared observations by Stein et al. (1969) it seems that the Loreta hypothesis (see O'Keefe, 1939) is true and together with Searle's abundance analysis one must conclude that these stars are shedding off mass into space and this mass is almost pure helium enriched with carbon. Maybe we should be careful with our conclusions until the story of these stars is revealed!

\section{Enrichment of the Interstellar Gas with Helium}

If one assumes that all stars somehow manage to become white dwarfs statistical arguments along the line of the early work by Temesvary and von Hoerner (1960) give an estimate of the mean helium content of the mass the more massive stars have to blow into space in order to settle down as white dwarfs. The mean mass $\bar{M}$ of the star which had time to become a white dwarf within the history of our galaxy is $\bar{M}=3.25 M_{\odot}$. Assuming the mean mass $\bar{M}_{w d}$ of a white dwarf to be $\bar{M}_{w d}=0.7 M_{\odot}$ the mean helium content $Y$ of the mass $\bar{M}-\bar{M}_{\text {wd }}$ which is ejected into interstellar space is

$$
Y=\frac{q \bar{M}}{\bar{M}-\bar{M}_{w d}}
$$

where $q \bar{M}$ is the amount of helium which goes into space. (In the numerical value of $\bar{M}$ there is assumed that all stars are formed at the beginning of the Galaxy. If this is not true then $\bar{M}$ is bigger which would decrease the value of $Y$. The formula given above is therefore an upper limit.) From the stellar evolution calculations it is indicated that $q=0.05$ is a reasonable value indicating that the helium content of the matter given back to the interstellar medium cannot account for the present helium content.

Truran et al. (1965) have made a more careful investigation. But they have overestimated the helium output by assuming that in the mass range of $1 M_{\odot}$ there are thick helium shells produced by the reactivation of the hydrogen burning shell which starts to produce helium again when from the interior the helium burning shell is approaching. But as can be seen from more recent model calculations, the effect of the reignition of hydrogen does not increase the total amount of helium. Quite to the contrary, the two shells are approaching each other leaving less and less helium 
in between. But even with this strong overestimate the authors found that within the lifetime of our Galaxy not enough helium can have been formed in order to account for the observed helium content.

\section{Other Mechanisms of Helium Production}

Since normal stellar evolution does not produce enough helium we might look for other processes. Tayler (1967) reports of a suggestion by Ledoux : The critical mass for vibrational stability of a homogeneous star on the main sequence is roughly given by

$$
M_{\text {crit }}=\frac{17}{\mu^{2}} M_{\odot}
$$

Stars above this mass are pulsationally unstable because of the $\varepsilon$-mechanism. One might assume that such a star evolves completely mixed - which because of the convective inner region surrounded by semi-convective layers is not too unreasonable. Then during the evolution when the molecular weight $\mu$ increases due to enrichment of helium the critical mass becomes smaller. If we imagine a star which starts on the main sequence with a mass above the critical mass it will pulsate and may shed mass into space (Appenzeller, 1970). For a pure hydrogen-helium mixture one has

$$
Y=\frac{8 \mu-4}{5 \mu}
$$

and therefore the critical mass and the mass $M_{\mathrm{He}}$ of helium in the star vary according to the following relations

$$
\mathrm{d} M_{\text {crit }}=-\frac{34}{\mu^{3}} \mathrm{~d} \mu, \quad \mathrm{d} M_{\mathrm{He}}=Y \mathrm{~d} M_{\text {crit }}=-34 \frac{8 \mu-4}{5 \mu^{4}} \mathrm{~d} \mu .
$$

Assuming that the mass of the star during its evolution is always just critical, the mass loss and the amount of helium lost while the mean molecular weight varies from the value $\mu=\frac{1}{2}$ for hydrogen to $\mu=\frac{4}{3}$ for helium can be obtained by integration. One finds that the star loses $58.5 \mathrm{M}_{\odot}$. The mean helium content of the matter blown into space then is $Y=0.42$ which could account for the observed helium content if most of the matter of the Galaxy has gone through vibrationally unstable massive stars.

There is always the possibility that helium is formed during the supernova process, in supermassive stars or in little bangs. If one insists in forming the helium after the big bang up to now, at least, one can make these objects responsible, since there is not enough knowledge about their physics in order to exclude them as effective helium producers.

\section{References}

Appenzeller, I.: 1970, Astron. Astrophys. 5, 355.

Christy, R. F.: 1966, Astrophys. J. 145, 340.

Christy, R. F.: 1968, Quart. J. Roy. Astron. Soc. 9, 13. 
Hayashi, C., Hoshi, R., and Sugimoto, D.: 1962, Progr. Theoret. Phys. Suppl. No. 22, 1.

Kippenhahn, R., Kohl, K., and Weigert, A.: 1967, Z. Astrophys. 66, 58.

Kippenhahn, R., Thomas, H.-C., and Weigert, A.: 1965, Z. Astrophys. 61, 241.

Lucy, L. B.: 1967, Astron. J. 72, 813.

O'Keefe, J. A.: 1939, Astrophys. J. 90, 294.

Paczynski, B. and Ziolkowski, J.: 1968, IAU Symp. No. 34, D. Reidel Publ. Co., Dordrecht-Holland, p. 396.

Roxburgh, I. W.: 1967, Nature 215, 838.

Searle, L.: 1961, Astrophys. J. 133, 531.

Stein, W. A., Gaustad, J. E., Gillet, F. C., and Knacke, R. F.: 1969, Astrophys. J. 155, L3.

Stobie, R. S.: 1969, Monthly Notices Roy. Astron. Soc. 144, 511.

Tayler, R. J.: 1967, Quart. J. Roy. Astron. Soc. 8, 313.

Temesvary, St. and v. Hoerner, S.: 1960, Z. Astrophys. 49, 30.

Thomas, H.-C.: 1967, Z. Astrophys. 67, 420.

Truran, J. W., Hansen, C. J., and Cameron, A. G. W.: 1965, Can. J. Phys. 43, 1616.

Weigert, A.: 1966, Z. Astrophys. 64, 395.

\section{DISCUSSION}

W. A. Fowler: It may be appropriate to point out that the production of helium has not only been thought to take place during the quasi-static stages of stellar evolution, but also during the final implosion-explosion stage, when the iron-to-helium phase change is so fast during implosion and the helium-to-iron build-up is so slow during the explosion. Eventually, quantitative calculations must be made concerning helium production during the final stages of stellar evolution.

I. Appenzeller: I would like to comment on the mechanism of pulsationally driven mass loss from vibrationally unstable very massive main-sequence stars. Based on non-linear pulsation calculations, I recently calculated rates for the pulsationally driven mass loss for zero-age main sequence stars of $60<M / M_{\odot}<600$. The main results of this investigation are: (a) vibrationally unstable stars with $M \leqslant 100 M_{\odot}$ do not lose any mass by this mechanism; (b) Stars with $M>300 M_{\odot}$ lose most of their initial mass on a time scale which is much shorter than the phase of hydrogen burning. Thus, most of the matter ejected from stars this massive has almost the initial chemical composition and is not much enriched in hydrogen. (c) Stars of about $100-300 M_{\odot}$, according to my calculations, may lose a considerable amount of helium-enriched matter. But, at least at the present stage of the evolution of our galaxy, stars in the mass range 100 to $300 M_{\odot}$ seem to be very rare objects.

J.P. Cox: I would like to ask Dr Appenzeller if he is aware of the work of K. Ziebarth and R. Talbot, who performed similar non-linear pulsation calculations on massive main sequence stars, and who, to the best of my knowledge, found no conclusive evidence of mass loss from this kind of mechanism?

I. Appenzeller: I am aware of the work by $\mathrm{K}$. Ziebarth from a preprint $\mathrm{I}$ have seen recently. Dr Ziebarth's result for a $100 M_{\odot}$ star seems to agree with my results, since he also does not find pulsationally driven mass loss for this mass value. I can not comment on the work of $\mathrm{R}$. Talbot since I do not know it.

St. Temesvary: I want to thank Dr Kippenhahn for mentioning our old paper, although old papers, like old ladies, do not necessarily enjoy to be reminded of their age. The point I wish to stress is that the amount of helium in the interstellar gas produced by stellar evolution still depends upon our knowledge about the rate of star formation in the early history of the Galaxy. To my knowledge, the value once derived by von Hoerner of a twenty to fifty times larger rate of star formation than today still stands. 